\title{
Sudden Cardiac Death and Sudden Cardiac Arrest in Patients with Human Immunodeficiency Virus: A Systematic Review
}

\author{
Basel Abdelazeem ${ }^{1}$, Kirolos Gergis $^{2}$, Nischit Baral ${ }^{1}$, Rohit Rauniyar ${ }^{3}$, Govinda Adhikari ${ }^{4}$
}

1. Internal Medicine, McLaren Health Care, Flint/Michigan State University (MSU), Flint, USA 2. Internal Medicine, McLaren Health Care, Flint, USA 3. Internal Medicine, McLaren Flint/Michigan State University (MSU), Flint, USA 4. Internal Medicine, McLaren Flint, Flint, USA

Corresponding author: Basel Abdelazeem, basel.abdelazeem@mclaren.org

\begin{abstract}
The importance of this review lies in its study of the risk of sudden cardiac death (SCD) and sudden cardiac arrest (SCA) in people living with the human immunodeficiency virus (PLWH). To the best of our knowledge, this is the first review investigating the effect of the human immunodeficiency virus (HIV) on SCD and SCA.

The review's objective was to determine the risk of SCD and SCA in PLWH. To do this, the electronic databases Ovid MEDLINE, EMBASE, Cochrane Central, Scopus, and Google Scholar were systematically searched to identify eligible studies published before January 31, 2021. Reference lists of the included studies were searched for further identification of relevant studies. The search terms included: "Sudden Cardiac Death," "Sudden Cardiac Arrest," "Human Immunodeficiency virus," "HIV," "Acquired immunodeficiency syndrome," and "AIDS." Only observational studies that assessed the association between SCD and SCA in PWLH were selected.
\end{abstract}

Data were extracted by two independent authors who screened titles, abstracts, and articles to meet the inclusion criterion. Quality assessment was done by using modified Downs and Black checklist.

A total of seven studies were included in this review. Five studies revealed a higher incidence of SCD in PLWH, two of which focused on patients with HIV and low left ventricular ejection fraction (LVEF). The other two studies were about the association of HIV and SCA. Studies reported that PLWH had a three- to five-fold higher incidence of SCD as compared to non-HIV patients. HIV patients with low LVEF had a higher incidence of SCD than HIV patients with normal LVEF. PLWH had a higher incidence of SCA and less successful cardiopulmonary resuscitation (CPR) as compared to patients without HIV. After adjusting for various confounders in multiple studies, all the studies reported a higher incidence of SCD in PLWH.

To conclude, PLWH is at an increased risk of SCD and SCA. Some risk factors for this include LVEF, viral load (VL), and the cluster of differentiation 4 (CD4) count. There is a paucity of data on the mechanisms involved, although a higher prevalence of cardiac fibrosis and interstitial fibrosis in PLWH may play a role. Because of the general suboptimal quality of the heterogeneous nature of the current evidence, further, rigorous studies are needed to determine the association of increased risk of SCD and SCA in PLWH.

Categories: Cardiology, Internal Medicine, HIV/AIDS

Keywords: sudden death, cardiac arrest, heart failure, systematic review, acquired immune deficiency syndrome (aids), hiv

\section{Introduction And Background}

Human immunodeficiency virus (HIV) infection was defined by the World Health Organization (WHO, 2007) as positive HIV antibody testing using rapid or laboratory-based enzyme immunoassay and/or positive HIVribonucleic acid (RNA) or HIV-deoxyribonucleic acid (DNA) or HIV p24 antigen. The result should be confirmed by a second test [1]. In 2019, 38 million people were living with HIV (PLWH), with around 690,000 deaths from acquired immunodeficiency syndrome (AIDS)-related illnesses worldwide. In 2019, around 2.2 million people were living with HIV in western and central Europe, and North America, with 12,000 HIVrelated deaths [2].

Sudden cardiac death (SCD) is a sudden unexpected cessation of cardiac function with hemodynamic collapse, which results in death within one hour of onset. If the patient survived, that event was called sudden cardiac arrest (SCA). In general, most studies reported that the incidence of SCD is 1 or 2 per 100,000 persons per year; however, Couper et al. reported that the incidence is between 0.75 and 11.9 cases per 100,000 persons per year [3]. The underlying etiology and pathogenesis of SCD vary; ventricular fibrillation is the most common cause of SCD. Structural heart disease, including ischemic heart disease, nonischemic cardiomyopathy, and noncardiac conditions like pulmonary embolism, aortic rupture, and other arrhythmias, are also associated with SCD [4]. Few studies reported an association between SCD and HIV. Therefore, in the present study, we performed a systemic review of whether HIV is associated with SCD and SCA, focusing on patients with heart failure.

\section{Review}

Methods

Data Sources and Search Strategy 
This systematic review was conducted according to Preferred Reporting for Systematic Review and MetaAnalysis (PRISMA) as recommended by Cochrane Collaboration [5]. A systematic literature review using MEDLINE, EMBASE, Cochrane Central, Scopus, and Google Scholar was performed using the terms "HIV," "AIDS," "Sudden Cardiac Death," and "Sudden Cardiac Arrest" for literature published till January 31, 2021. We included additional articles found in the review of bibliographies or suggested by co-authors based on their relevance to the selected search terms.

Study Selection and Eligibility Criteria

Search results were saved in EndNote files and transferred into Covidence. Two reviewers (BA and NB) independently performed the title and abstract screening to include the articles regarding PLWH, SCD, and SCA. Conflicts were resolved through a third author (KG). Next, we reviewed the selected articles using the following inclusion criteria: randomized controlled trial (RCT); observational study; study population involving adults with HIV; primary outcome SCD, SCA; assessment of incidence and prevalence between HIV and SCD or SCA; English written text; Full text. We excluded case reports and case series with five or fewer cases, studies population children, non-full text articles. Conflicts were resolved through the third author (KG)

Data Extraction

Data from included studies were extracted independently by two reviewers (BA, KG) from Covidence. The consensus was reached in case of any inconsistency with a third author (NB). The data extracted for qualitative synthesis included location, year of study, study design, sample size, population age (in years), and incidence or prevalence of SCD or SCA. The data were entered in Microsoft Office Excel 2016 (Microsoft Corporation, Redmond, WA) by BA and checked by a second author (KG). Any discrepancy was resolved by a discussion between the authors.

Assessment of the Methodological Quality of Included Reviews

We assessed the methodical rigor of the included studies using the modified Downs and Black checklist for RCTs and non-randomized studies [6]. The checklist has 27 items with a total possible score of 28 . Papers were rated excellent if they scored above 25 , good if they scored between 20 and 25 , fair if they scored between 15 and 19, and poor if they scored <15. Each study was assessed by two independent investigators (NB and RR), and discrepancies in scoring were resolved by a third author (BA).

Analysis of Outcome

The primary outcome of interest was the association between SCD and HIV patients with and without heart failure (HF). The secondary outcome was the association between HIV and SCA.

\section{Result}

Study Identification and Selection

We identified 272 relevant citations. After removing the duplicates, 140 citations were selected as potentially relevant. The titles and abstracts were screened, and then 10 full-text articles were chosen for further review. Three articles were excluded, one of them was an editorial review and the other two were irrelevant intervention and outcome. Finally, seven articles were included in this systematic review (Figure 1).

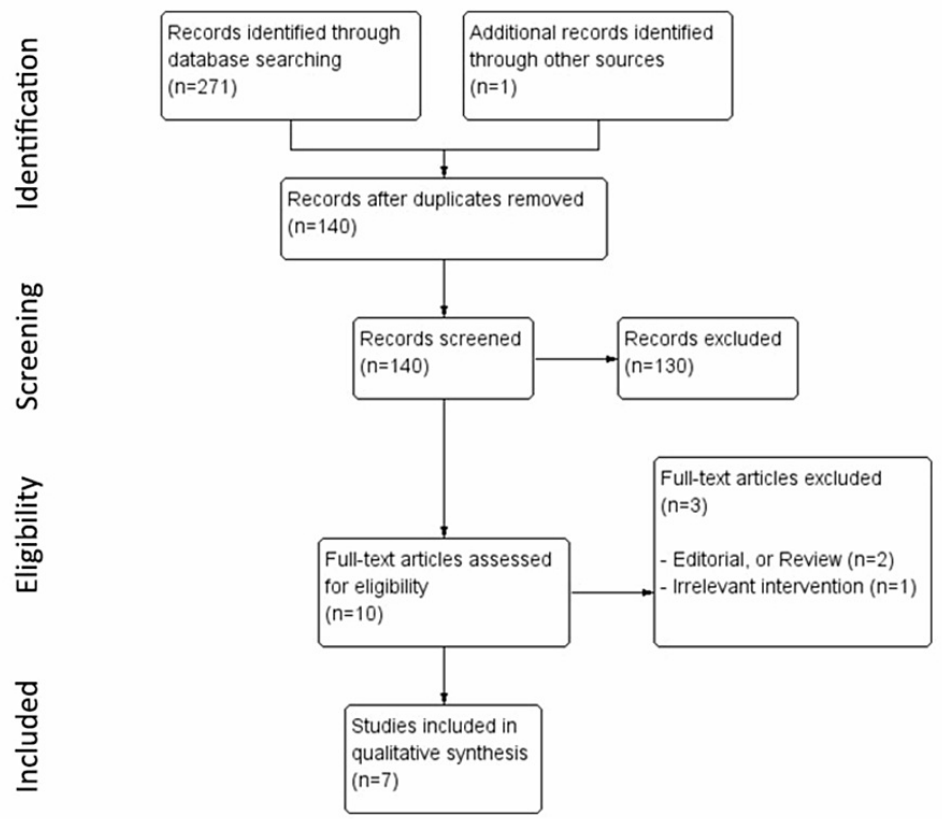

FIGURE 1: Flow diagram of the study retrieved, excluded, assessed, and 


\section{Cureus}

included

Characteristics of Included Studies

The patient's baseline characteristics and summary of the included studies are outlined (Table 1 and Table 2). The patients' baseline characteristics included age, gender, body mass index (BMI), HIV parameters, risk factors, and co-morbidities. 


\section{Cureus}

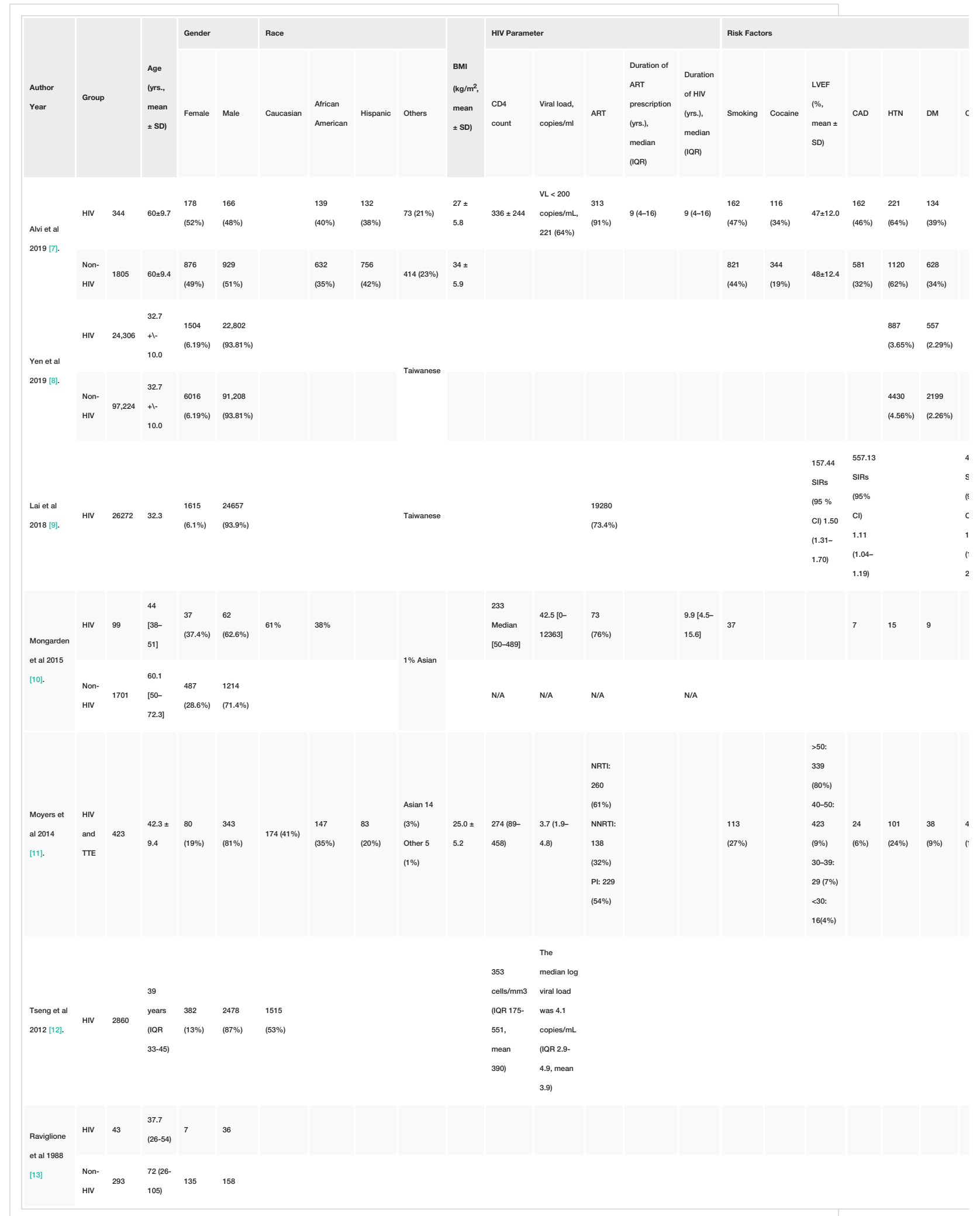

\section{TABLE 1: Baseline characteristics}

HIV: human immunodeficiency virus; BMI: body mass index; CD: cluster of differentiation; ART: antiretroviral therapy; LVEF: left ventricular ejection fraction; CAD: coronary artery disease; HTN: hypertension; DM: diabetes mellitus; CKD: chronic kidney disease; SIRs: systemic inflammatory response syndrome; NRTI: nucleoside reverse-transcriptase inhibitor; NNRTI: non-nucleoside reverse-transcriptase inhibitor; IQR: interquartile range

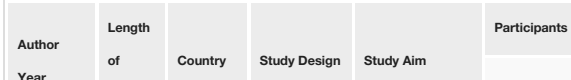




\section{Cureus}

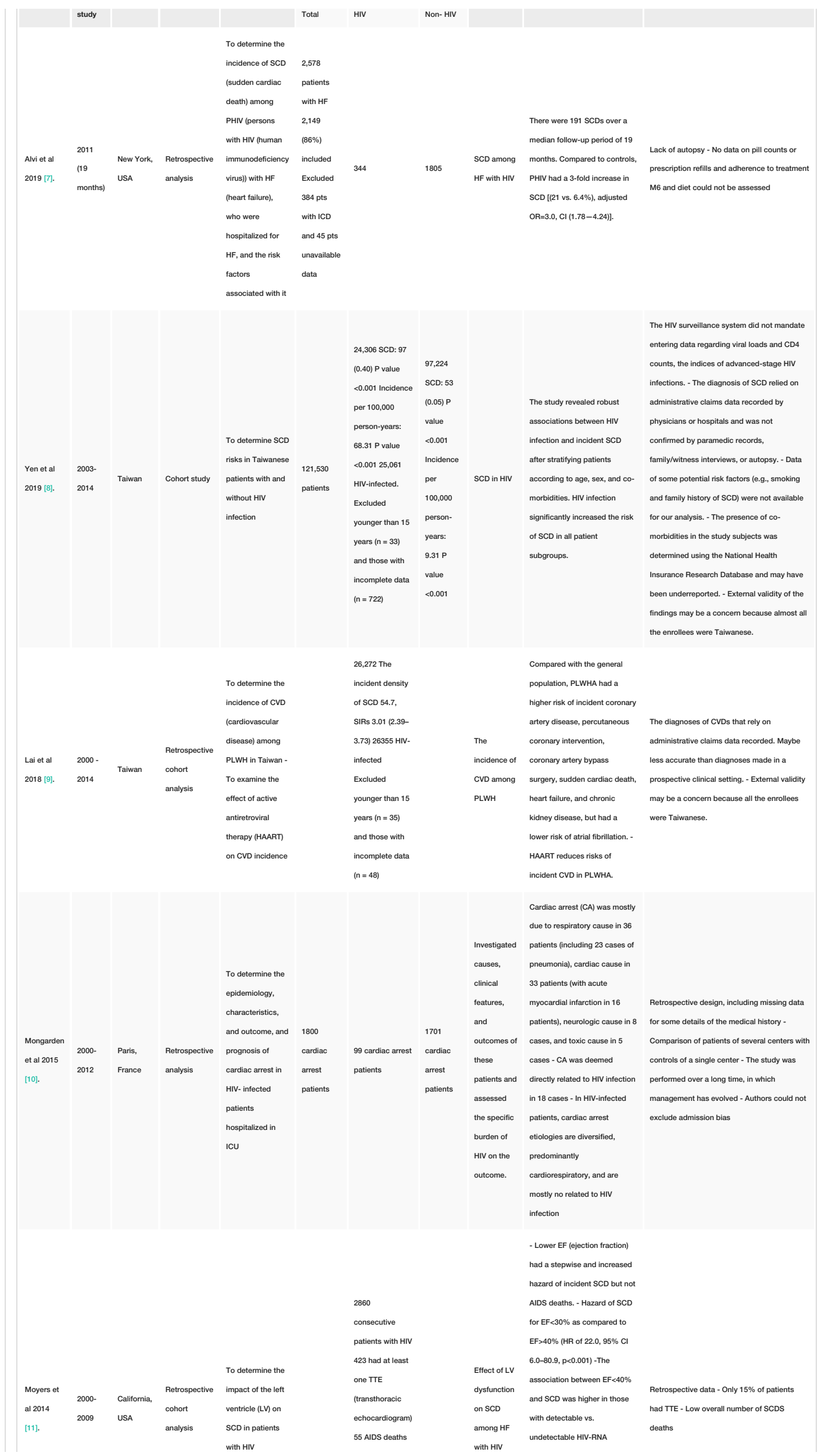




\section{Cureus}

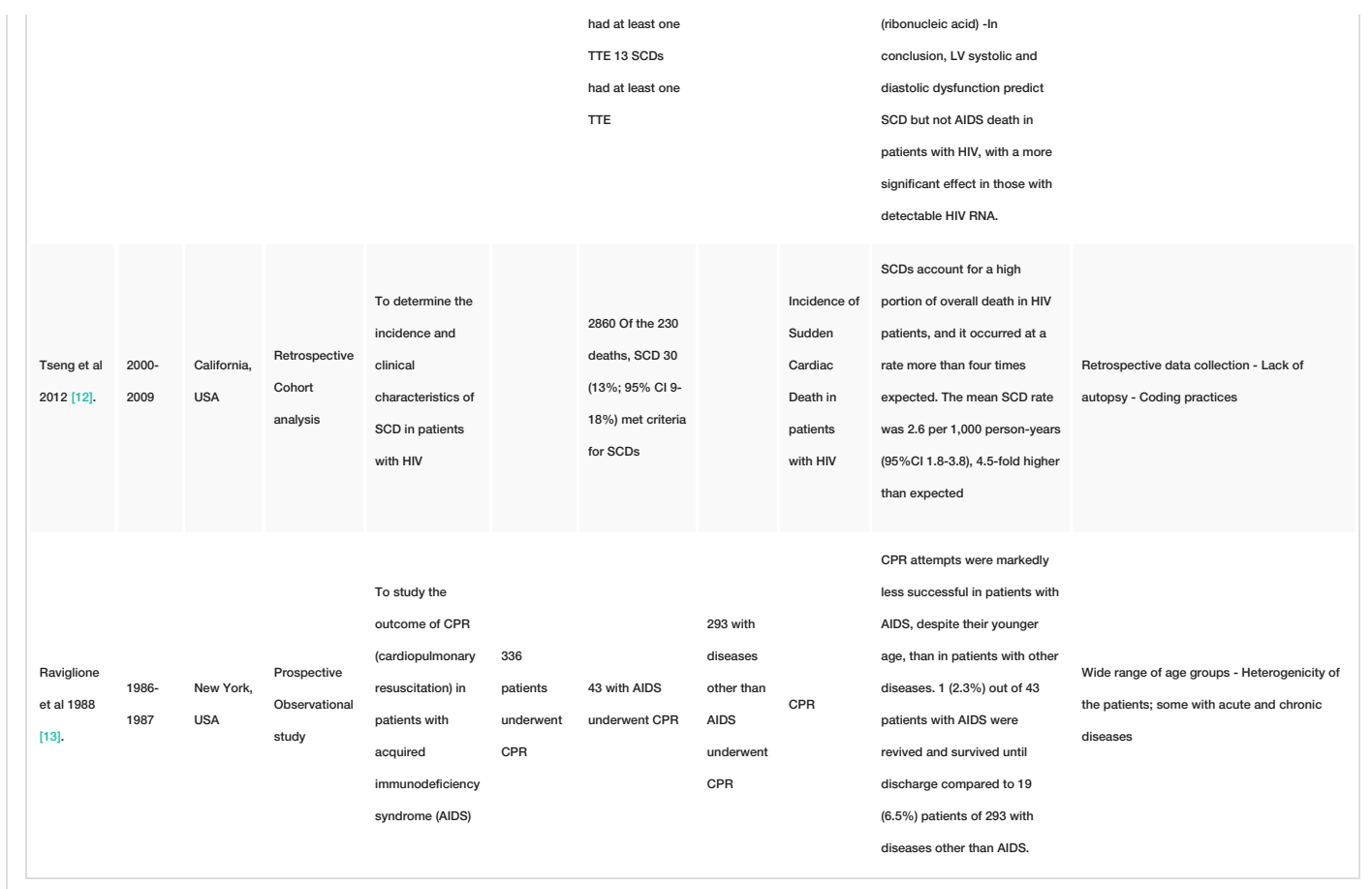

TABLE 2: Literature summary

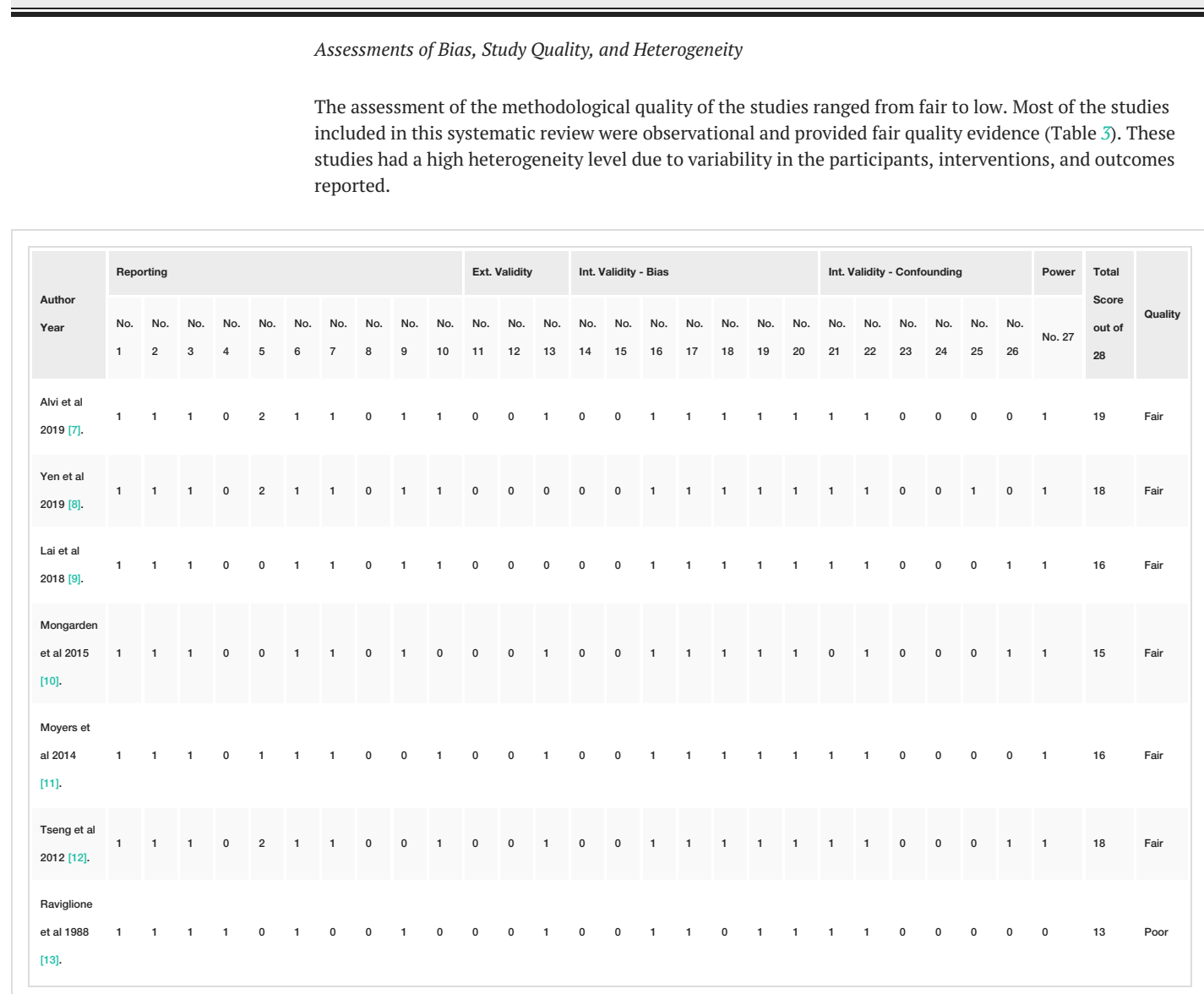

TABLE 3: Modified Down and Black checklist

Primary Outcome

Our primary outcome of interest was the risk of SCD or SCA in PWLH. 
Our secondary outcome was the risk of SCD or SCA in PWLH and having low LVEF and the success rate of CPR in PLWH.

\section{Discussion}

SCA leading to SCD is the most common cause of death worldwide, representing more than $50 \%$ of all cardiovascular disease deaths [14]. Myocardial fibrosis, primary electrophysiological conditions, and coronary artery disease predispose to SCD. Possible precipitating factors include ischemia, hypoxia, toxins, and electrolyte abnormalities. Combination of susceptible myocardium and precipitating factors lead to ventricular arrhythmias and SCA, followed by SCD if the CPR was unsuccessful in retrieving the patient.

In this systematic review, we included seven studies that reported the effect of HIV on SCD; five studies analyzed the association between SCD and HIV; Yen et al. [8] reported a higher incidence rate of SCD in PLWH than non-HIV patients, 68.31 and 9.31 per 100,000 persons per year, respectively. Lai et al. [9] reported that compared with the general population, PLWH had a higher risk of incident coronary artery disease, percutaneous coronary intervention, coronary artery bypass surgery, SCD, heart failure, and chronic kidney disease but had a lower risk of atrial fibrillation. Tseng et al. [12] reported that SCD account for most cardiac and many non-AIDS natural deaths in PLWH. Alvi et al. [7] and Moyers et al. [11] were mainly on Heart Failure (HF) patients and determined the impact of LV dysfunction on SCD in PLWH. Alvi et al. [7] reported that PLWH had a 3-fold increase in SCD [(21 vs. 6.4\%), adjusted OR=3.0, CI (1.78-4.24)]. Moyers et al. [11] reported that the incidence of $\mathrm{SCD}$ for $\mathrm{EF}<30 \%$ as compared to $\mathrm{EF}>40 \%$ was significantly higher (HR of $22.0,95 \%$ CI $6.0-80.9, \mathrm{p}<0.001)$.

Tseng et al. [12] involved 2860 HIV patients in the study; out of 230 deaths over 3.7 median years of followup, 30 patients met the criteria for SCD (13\%; 95\% CI 9-18\%). SCD accounted for 86\% (30/35) of all cardiac deaths. They reported that the mean SCD rate was 2.6 per 1,000 person-years (95\% CI 1.8-3.8), 4.5 -fold higher than expected. Yen et al. [8] also reported a higher incidence of SCD in HIV patients. The study included 121,530 patients (24,306 PLWH and 97,224 matched controls), from those 5342 (4.40\%) of died; among them, 150 (0.12\%) died of SCD. Among 150 SCD events, 97 (64.7\%) and 53 (33.3\%) occurred in PLWH and controls, respectively, which corresponded to incidences of 68.31 in PLWH and 9.31 per 100,000 personyears in controls $(\mathrm{P}<0.001)$. Lai et al. [9] also looked for CVD in HIV patients and reported an incident density of 54.7, Standardized incidence rate (SIR) 3.01 (95\% CI 2.39-3.73) for SCD in the subgroup analysis. All three articles agreed that the PLWH has a higher incidence of SCD than the general population, and it was statistically significant.

Both Alvi et al. [7] and Moyers et al. [11] were interested in the HIV patient who had HF or LV dysfunction. Alvie et al. [7] studied 2,578 HF hospitalized patients; 344 were PLWH. And they subclassified the patient according to the LVEF $<35 \%, 35-49 \%,>50 \%$. 191 SCDs occurred over 19 months of the follow-up period and reported that the rate of SCD was higher among PLWH compared to non-HIV infected individuals (21 vs. $6.4 \%, \mathrm{p}<0.001)$ [adjusted OR=3.0, CI $=(1.78-4.24)$ ]. They also reported that low LVEF, low CD4, and higher viral load were predictors of SCD. Moyer et al. [11] determined the impact of LVEF on SCD in PLWH. They included 423 patients who had at least one transthoracic echocardiogram, and the results revealed that the lower the EF, the higher the risk of SCD. The risk of SCD for $\mathrm{EF}<30 \%$ was higher compared to $\mathrm{EF}>40 \%$ (HR of $22.0,95 \% \mathrm{CI} 6.0-80.9, \mathrm{p}<0.001)$. Both studies showed that HIV patients with HF have a higher rate of SCD than HIV patients without HF, and the lower the EF, the higher the SCD events.

Two studies analyzed the association between SCA and HIV, Mongaeden et al. [10] described the burden of HIV on cardiac arrest by comparing 99 HIV to 1701 non-HIV patients with a primary outcome of determining CA characteristics in HIV patients in ICU from 2000-2012 in France. They reported that out of the 99 HIV patients, 33 patients sustained cardiac arrest, out of which 18 were directly related to HIV infection. Raviglione et al. [13] studied the association between CPR and HIV. They compared 43 HIV patients to 293 Non-HIV, with the primary outcome of the success of CPR. The results revealed that CPR attempts were markedly less successful in a patient with HIV.

This study has many limitations. First, the quality of included studies was limited to fair, with one poor article. Variation in study quality contributed to the heterogeneity of findings noted. Other sources of heterogeneity are likely to include population differences, including age as a factor contributing to SCD or SCA. Second, all of the included studies were observational studies (prospective or retrospective) affecting the extracted data. Some medical history details can be missed, which leads to selection and information biases. Third, all of the articles lacked the autopsy findings for the SCD cases, so all cases of SCD were presumed not confirmed. Usually, a histopathological exam of the heart is essential to know the underlying etiology [15]. Fourth, Sudden death in PLWH with CD4+ T cell count $<50 / \mathrm{cm}$ has been termed as HIV death rather than SCD. The cause of death in such circumstances is severe immunodeficiency rather than SCD. Alvi et al. [7], Mongarden et al. [10], Moyers et al. [11], and Tseng et al. [12] reported that their patients had a CD4+ T cell count of more than 50/cm. But Yen et al. [8], Lai et al. [9], and Raviglione et al. [13] didn't report $\mathrm{CD} 4+\mathrm{T}$ cell count for their patients. Fifth, external validation was questionable in all of these studies, as these studies were in a specific location and lacked randomization.

To the best of our knowledge, this is the first review investigating the effect of HIV on SCD and SCA.

\section{Conclusions}

This systematic review revealed a higher rate of SCD and SCA in a patient with HIV than in the general population. The risk is even higher in HIV patients with low LVEF. After adjusting for various confounders like age, sex, and race, the risk factors for cardiovascular disease, persistently low CD4 counts, and high viral loads are also associated with increased risk of SCA and SCD in PLWH. There is a paucity of data on the 
mechanisms involved, although the prevalence of cardiac fibrosis and interstitial fibrosis in PLWH may play a role. Because of the general suboptimal quality of the heterogeneous nature of the current evidence, further rigorous studies are needed to determine the association of increased risk of SCD and SCA in PLWH.

\section{Additional Information}

\section{Disclosures}

Conflicts of interest: In compliance with the ICMJE uniform disclosure form, all authors declare the following: Payment/services info: All authors have declared that no financial support was received from any organization for the submitted work. Financial relationships: All authors have declared that they have no financial relationships at present or within the previous three years with any organizations that might have an interest in the submitted work. Other relationships: All authors have declared that there are no other relationships or activities that could appear to have influenced the submitted work.

\section{Acknowledgements}

We thank Sarah Ayad, MD, for her valuable assistance in data acquisition and table formatting. We also thank Sandi Banour, Pharm.D., BSPH, for her excellent efforts in editing and reviewing the final draft.

\section{References}

1. WHO case definitions of HIV for surveillance and revised clinical staging and immunological classification of HIV-related disease in adults and children. (2007). https://apps.who.int/iris/bitstream/handle/10665/43699/9789241595629_eng.pdf.

2. Joint United Nations Programme on HIV/AIDS (UNAIDS) fact sheet: World AIDS Day 2019-global HIV statistics. (2020). https://www.unaids.org/sites/default/files/media_asset/UNAIDS_FactSheet_en.pdf.

3. Couper K, Putt O, Field R, et al.: Incidence of sudden cardiac death in the young: a systematic review . BMJ Open. 2020, 10:040815. 10.1136/bmjopen-2020-040815

4. Estes NA 3rd: Predicting and preventing sudden cardiac death. Circulation. 2011, 124:651-656. 10.1161/CIRCULATIONAHA.110.974170

5. Moher D, Liberati A, Tetzlaff J, et al.: Preferred reporting items for systematic reviews and meta-analyses: the PRISMA statement. PLoS Med. 2009, 6:1000097. 10.1371/journal.pmed.1000097

6. Downs SH, Black N: The feasibility of creating a checklist for the assessment of the methodological quality both of randomised and non-randomised studies of health care interventions. J Epidemiol Community Health. 1998, 52:377-384. 10.1136/jech.52.6.377

7. Alvi RM, Neilan AM, Tariq N, et al.: The risk for sudden cardiac death among patients living with heart failure and human immunodeficiency virus. JACC Heart Fail. 2019, 7:759-767. 10.1016/j.jchf.2019.04.025

8. Yen YF, Lai YJ, Chen YY, et al.: Association of HIV infection and antiretroviral therapy with sudden cardiac death. J Acquir Immune Defic Syndr. 2019, 82:468-474. 10.1097/QAI.0000000000002161

9. Lai YJ, Chen YY, Huang HH, Ko M-C, Chen C-C, Yen Y-F: Incidence of cardiovascular diseases in a nationwide HIV/AIDS patient cohort in Taiwan from 2000 to 2014. Epidemiol Infect. 2018, 146:2066-2071. 10.1017/S0950268818002339

10. Mongardon N, Geri G, Deye N, et al.: Etiologies, clinical features and outcome of cardiac arrest in HIVinfected patients. Int J Cardiol. 2015, 201:302-307. 10.1016/j.ijcard.2015.08.055

11. Moyers BS, Secemsky EA, Vittinghoff E, Wong JK, Havlir DV, Hsue PY, Tseng ZH: Effect of left ventricular dysfunction and viral load on risk of sudden cardiac death in patients with human immunodeficiency virus. Am J Cardiol. 2014, 113:1260-1265. 10.1016/i.amicard.2013.12.036

12. Tseng ZH, Secemsky EA, Dowdy D, et al.: Sudden cardiac death in patients with human immunodeficiency virus infection. J Am Coll Cardiol. 2012, 59:1891-1896. 10.1016/j.jacc.2012.02.024

13. Raviglione MC, Battan R, Taranta A: Cardiopulmonary resuscitation in patients with the acquired immunodeficiency syndrome. A prospective study. Arch Intern Med. 1988, 148:2602-2605.

14. Zipes DP, Camm AJ, Borggrefe M, et al.: ACC/AHA/ESC 2006 guidelines for management of patients with ventricular arrhythmias and the prevention of sudden cardiac death-executive summary: a report of the American College of Cardiology/American Heart Association Task Force and the European Society of Cardiology Committee for Practice Guidelines (Writing Committee to Develop Guidelines for Management of Patients with Ventricular Arrhythmias and the Prevention of Sudden Cardiac Death) developed in collaboration with the European Heart Rhythm Association and the Heart Rhythm Society. Eur Heart J. 2006, $27: 2099-2140.10 .1093 /$ eurheartj/ehl199

15. de Noronha SV, Behr ER, Papadakis M, et al.: The importance of specialist cardiac histopathological examination in the investigation of young sudden cardiac deaths. Europace. 2014, 16:899-907. 10.1093/europace/eut329 